\title{
THE ASYMPTOTIC BEHAVIOR OF NONLINEAR SCHRÖDINGER EQUATIONS
}

\author{
BY YOSHIO TSUTSUMI AND KENII YAJIMA
}

We consider the nonlinear Schrödinger equation with power interactions

$$
i \partial u / \partial t=-\frac{1}{2} \Delta u+\lambda|u|^{p-1} u
$$

in $\mathbf{R}^{n}, n \geq 2$, with $\lambda>0$. Proposing a new method for studying the large time behavior of the solutions of (NS), we prove the following theorem. $H_{0}=-\frac{1}{2} \Delta$ is the free Hamiltonian and

$$
\Sigma=\left\{u \in L^{2}\left(\mathbf{R}^{n}\right) ;\|u\|_{2}+\|\nabla u\|_{2}+\|x u\|_{2}<\infty\right\},
$$

where $\|u\|_{q}$ denotes the $L^{q}$-norm of $u$.

Theorem. Let $1+2 / n<p<1+4 /(n-2)$. Then for any $u_{0} \in \Sigma$ there exists a unique $u_{ \pm} \in L^{2}\left(\mathbf{R}^{n}\right)$ such that the solution $u(t)$ of (NS) with $u(0)=u_{0}$ has the free asymptote $u_{ \pm}$as $t \rightarrow \pm \infty$ :

$$
\lim _{t \rightarrow \pm \infty}\left\|u(t)-\exp \left(-i t H_{0}\right) u_{ \pm}\right\|_{2}=0 \text {. }
$$

REMARK. Since it is shown by Glassey [4] and Strauss [6] that if $1<p \leq$ $1+2 / n$ any nontrivial solution $u(t)$ of (NS) with $u(0) \in S$ never satisfies (2), our theorem achieves the least possible exponent $1+2 / n$ for this direction.

In the sequel we shall prove the theorem. Our proof is based on the following observation: Since the asymptotic profile of the free evolution $\exp \left(-i t H_{0}\right) f$ is given by $(1 / i t)^{n / 2} \exp \left(i x^{2} / 2 t\right) \hat{f}(x / t)$ and (NS) is transformed by the conjugation $C$,

$$
u(t, x)=(C v)(t, x)=(1 / i t)^{n / 2} \exp \left(i x^{2} / 2 t\right) \overline{v(1 / t, x / t)},
$$

into the new equation

$$
i \partial v / \partial t=-\frac{1}{2} \Delta v+\lambda|t|^{n(p-1) / 2-2}|v|^{p-1} v,
$$

the relation (2) is equivalent to the existence of

$$
\lim _{t \rightarrow \pm 0} v(t) \equiv v_{ \pm}(0) \text { in } L^{2}\left(\mathbf{R}^{n}\right) \text {. }
$$

Here and hereafter $\hat{f}$ and $\check{f}$ are the Fourier transform of $f$ and the inverse Fourier transform of $f$, respectively. The equation (TNS) has almost the same form as (NS) and, for $p>1+2 / n, t^{n(p-1) / 2-2}$ is integrable near $t=0$. Thus we expect the existence of the limit (4) for those $p$ 's.

The equation (NS) has interested many authors and there is quite a body of literature. Among them, we mention the following which are related to

Received by the editors September 14, 1983.

1980 Mathematics Subject Classification. Primary 35J60, 81C05.

(c) 1984 American Mathematical Society $0273-0979 / 84 \$ 1.00+\$ .25$ per page 
our result. For $1 \leq p<1+4 /(n-2)$, the global existence of the solution $u(t)$ of (NS) with $u(0) \in H^{1}\left(\mathbf{R}^{n}\right)$ is proved by Ginibre and Velo [1]. In [2] they also show the above theorem for $1+4 / n<p<1+4 /(n-2)$ (see also Lin and Strauss [5]). The lower exponent $1+4 / n$ is subsequently decreased to $\gamma(n)=\left(n+2+\sqrt{n^{2}+12 n+4}\right) / 2 n$ in Strauss [7], but the allowed $u(0)$ are restricted to be small in a certain norm.

Proof. From [1] and [2] we already know that (NS) has a unique global solution $u(t, \cdot) \in C\left(\mathbf{R}^{1} ; \Sigma\right)$ with $u(0)=u_{1}$. We note that the solution of (NS) means the so-called mild solution of the integral equation associated with the differential equation (NS) (see [1]). Then a direct computation shows that $v(t)=\left(C^{-1} u\right)(t) \in C\left(\mathbf{R}^{ \pm} ; \Sigma\right)$ is a unique solution of (TNS).

We prove the theorem for $t \rightarrow+\infty$ with $1+2 / n<p \leq 1+4 / n$ only. The other cases may be proved similarly. We first obtain two conservation laws for (TNS). We multiply (TNS) by $t^{2-n(p-1) / 2} \partial \bar{v} / \partial t$ and take the real part. This leads us to

$$
\begin{aligned}
t^{2-n(p-1) / 2} & \|\nabla v(t)\|_{2}^{2}+\frac{4}{p+1} \int_{\mathbf{R}^{n}}|v(t, x)|^{p+1} d x \\
\geq & s^{2-n(p-1) / 2}\|\nabla v(s)\|_{2}^{2}+\frac{4}{p+1} \int_{\mathbf{R}^{n}}|v(s, x)|^{p+1} d x
\end{aligned}
$$

for all $0<s \leq t<+\infty$. We note that this rather formal calculation can be easily justified by the regularizing technique of Ginibre and Velo [1]. Next we multiply (TNS) by $\bar{v}$ and take the imaginary part to obtain

$$
\|v(t)\|_{2}=\|v(s)\|_{2}, \quad 0<s \leq t<+\infty .
$$

By (5) and (6) we conclude that

$$
t^{2-n(p-1) / 2}\|\nabla v(t)\|_{2}^{2}<C_{1}, \quad\|v(t)\|_{p+1}<C_{2}, \quad\|v(t)\|_{2}<C_{3}
$$

for all $t \in(0,1]$, where $C_{1}, C_{2}$ and $C_{3}$ depend only on $\|v(1)\|_{H^{1}}$ and $\|v(1)\|_{p+1}$. Let $\varphi \in H^{1}\left(\mathbf{R}^{n}\right)$. By (TNS),

$$
\begin{aligned}
(v(t)-v(s), \varphi)= & \int_{s}^{t}\left(\frac{\partial v(\tau)}{\partial \tau}, \varphi\right) d \tau \\
= & -\frac{i}{2} \int_{s}^{t}(\nabla v(\tau), \nabla \varphi) t \tau \\
& -i \int_{s}^{t} \tau^{n(p-1) / 2-2}\left(|v(\tau)|^{p-1} v(\tau), \varphi\right) d \tau
\end{aligned}
$$

for $0<t, s<+\infty$, where $(\cdot, \cdot)$ is the inner product in $L^{2}\left(\mathbf{R}^{n}\right)$. Since $n(p-1) / 2-2>-1$ for $p>1+2 / n$ and $H^{1}\left(\mathbf{R}^{n}\right)$ is dense in $L^{2}\left(\mathbf{R}^{n}\right),(7)$ and (8) show that the weak limit

$$
\underset{t \rightarrow+0}{\mathrm{w}-\lim } v(t) \equiv v(0)
$$

exists in $L^{2}\left(\mathbf{R}^{n}\right)$. Now choose $\varphi=v(t)$ in (8). Then

$$
\begin{aligned}
|(v(t)-v(s), v(t))| \leq & \frac{1}{2} \int_{s}^{t}\|\nabla v(\tau)\|_{2} d \tau \cdot\|\nabla v(t)\|_{2} \\
& +\int_{s}^{t} \tau^{n(p-1) / 2-2}\|v(\tau)\|_{p+1}^{p} d \tau \cdot\|v(t)\|_{p+1}
\end{aligned}
$$


for all $0<s \leq t<+\infty$. Applying (7) to (10), we have

$$
\begin{array}{r}
|(v(t)-v(s), v(t))| \leq C_{4}\left[\frac{4}{n(p-1)}\left\{t^{n(p-1) / 2-1}-s^{n(p-1) / 4} t^{n(p-1) / 4-1}\right\}\right. \\
\left.\quad+\frac{2}{n(p-1)-2}\left\{t^{n(p-1) / 2-1}-s^{n(p-1) / 2-1}\right\}\right] .
\end{array}
$$

Let $s \rightarrow+0$ and use (9) to obtain

$$
|(v(t)-v(0), v(t))| \leq C_{5} t^{n(p-1) / 2-1}
$$

with $C_{5}>0$ depending only on $n, p,\|v(1)\|_{p+1}$ and $\|v(1)\|_{H^{1}}$. Therefore,

$$
\begin{aligned}
\|v(t)-v(0)\|_{2}^{2} & =(v(t)-v(0), v(t))-(v(t)-v(0), v(0)) \\
& \leq C_{5} t^{n(p-1) / 2-1}+|(v(t)-v(0), v(0))| \\
& \rightarrow 0 \quad(t \rightarrow+0) .
\end{aligned}
$$

Returning to (NS) we see that

$$
\left\|\exp \left(-i t H_{0}\right) \check{\bar{v}}(0)-u(t)\right\|_{2} \rightarrow 0 \quad(t \rightarrow+\infty)
$$

as desired.

The construction of wave operators and the asymptotic completeness problem will be discussed elsewhere.

\section{REFERENCES}

1. J. Ginibre and G. Velo, On a class of nonlinear Schrödinger equations. I: The Cauchy problem, J. Funct. Anal. 32 (1979), 1-32.

2. , On a class of nonlinear Schrödinger equations. II: Scattering theory, J. Funct. Anal. 32 (1979), 33-71.

3. - Sur une équation de Schrödinger non linéaire avec interaction non locale, Nonlinear Partial Differential Equations and Their Applications, Collège de France Seminair, Vol. II, Pitman, Boston, 1981.

4. R. T. Glassey, On the asymptotic behavior of nonlinear wave equations, Trans. Amer. Math. Soc. 182 (1973), 187-200.

5. J. E. Lin and W. A. Strauss, Decay and scattering of solutions of a nonlinear Schrödinger equation, J. Funct. Anal. 30 (1978), 245-263.

6. W. A. Strauss, Nonlinear scattering theory, Scattering Theory in Math. Physics, Reidel, Dordrecht, 1974, pp. 53-78.

7. __ Nonlinear scattering theory at low energy, J. Funct. Anal. 41 (1981), 110-133.

Department of Pure and Applied Sciences, University of Tokyo, 3-8-1 Komaba, MEguro-KU, TOKYo, 153 Japan 